Polymer Journal, Vol. 39, No. 5, pp. 464-470 (2007)

(C) 2007 The Society of Polymer Science, Japan

\title{
Hierarchically Structured Silica from Mediation of Linear Poly(ethyleneimine) Incorporated with Acidic/Basic Additives
}

\author{
Ren-Hua JIN ${ }^{\dagger}$ and Jian-Jun YUAN \\ Synthetic Chemistry Lab, Kawamura Institute of Chemical Research, 631 Sakado, Sakura 285-0078, Japan
}

(Received January 9, 2007; Accepted February 23, 2007; Published April 9, 2007)

\begin{abstract}
A unique hierarchically structured silica directed by simple hydrolytic condensation of tetramethoxysilane (TMOS) on aggregates of linear poly(ethylenemine) (LPEI) incorporated with acidic and basic additives was described. Interestingly, the shapes of the resulting silicas showed nanofiber-based plate, bundle, sphere, flower and stick upon the additives, such as $\mathrm{HCl}$, ammonia, Tetra(4-sulfophenyl)porphyrin (TSPP), bifunctional organic acids. All the additives are relations in the proton transfer with LPEI in aqueous media thus induce the corresponding aggregates of the LPEI. Silicification just occurs on the aggregates to give the structured silica. [doi:10.1295/polymj.PJ2006210] KEY WORDS Biomimetic Silica Synthesis / Biomineralization / Poly(ethyleneimine) / Porphyrin / Hierarchical Silica Structure / Nano Structured Silica /
\end{abstract}

In nature, organisms have succeeded in synthesizing the numerous biominerals that bear the unique and species-specific structures and properties through evolution. ${ }^{1,2}$ The biological production of inorganic structures or composites of organic and inorganic materials is biologically controlled, and generally accomplished under water environments and mild conditions. ${ }^{3}$ By mimicking the biomineralization process, various inorganic solids have been fabricated in laboratories under the high-degree control over the shapes and structures, such as the biomimetic morphosynthesis of metal carbonates/sulphates/phosphates. ${ }^{4,5}$ Moreover, bioinspired routes have also been developed for the controllable synthesis of the nanostructured metals, metal oxides and other semiconductor compounds. ${ }^{5,6}$ The biomimetic or bioinspired approaches to inorganic materials are of great interest to both academic research and technological applications.

Silica is widely applicable for catalysis, chemical and biological separations, photonic and electronic devices, bio-encapsulation and drug delivery, coatings, energy storage, and so on. ${ }^{7}$ In the past decade, numerous works appeared to fabricate silicas with controllable nanostructures and shapes, such as mesoporous silicas ${ }^{8,9}$ silica fibers/tube, ${ }^{10}$ silicas with shape character, ${ }^{11-13}$ and so on. ${ }^{14}$ However, these silica productions typically require high temperature or the use of caustic chemicals. By contrast, in the biological processing, silica formation occurs under mild conditions to give precisely controlled shapes and patterns, such as seen in diatoms and sponges ${ }^{15-18}$ Recently, based on the understanding of biosilicification, ${ }^{19,20}$ biomimetic synthesis of silica under ambient conditions has been accomplished by using a number of bio-derived or synthetic organic molecules. ${ }^{18,19,21-34}$

Cha et $a l .{ }^{33}$ used a series of diblock copolymers consisted of poly(lysine) and poly(cystein) blocks in silicification and found that the silicas with hard spheres or well-defined columns were controllable by switching $\mathrm{O}_{2}$ and $\mathrm{N}_{2}$ atmosphere in self-assembly of the polypeptides. Stone et al. ${ }^{35,36}$ and Clarson et $a l .{ }^{37}$ described the formation of complex silicas by applying physical fields, using additives or changing the size of polypeptides. Sumper ${ }^{38}$ showed the formation of roughly hexagonal silica structures when using polyamine-stabilized sol as silicon source in place of monosilicic acid. Very recently, we demonstrated that the multiply shaped silicas could be facilely obtained by the mediation of organized linear poly(ethyleneimine) (LPEI) aggregates that serve simultaneously three parts, such as catalyst, scaffold and template for the silica deposition. ${ }^{39-41}$ In aqueous media, LPEI forms easily hierarchical aggregates which are constructed by arrangement of unit structures consisting of crystalline fibrous core with brush-like ethyleneimine shell (see Scheme 1). ${ }^{39,41}$ These unique aggregates are responsible for the rapid silica deposition with controlled fibrous network from TMOS under mild conditions (Scheme 1). ${ }^{42}$

Aggregates of LPEI organized in neat water with

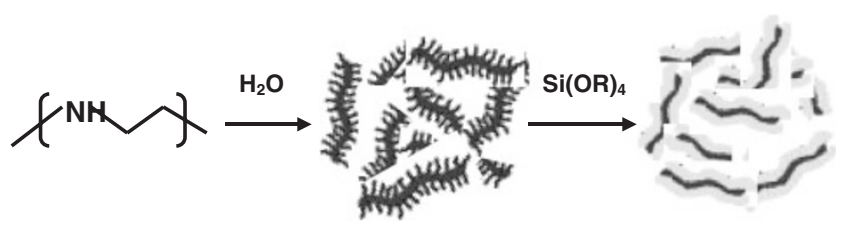

Scheme 1. Schematic representation of LPEI aggregation and silica deposition.

${ }^{\dagger}$ To whom correspondence should be addressed (Fax: +81-43-498-2202, E-mail: jin@kicr.or.jp). 
different concentrations are capable of directing nanofiber-based silica bundles, leaves and films. Furthermore, silicas with more complex shapes and structures, such as nanofiber-based asters, dendrimer, flower, nanosphere-based networks have been directed simply by changing architectures of LPEI or by adjusting aqueous media composition for LPEI organization. ${ }^{39-42}$ Typically, we have shown that flower-like silicas are directed by incorporation of a simple LPEI with acidic compound of tetra(sulfophenyl)porphyrin (TSPP). ${ }^{40}$ This indicates that additives that are capable of interacting with LPEI with proton transfer would be effective triggers to induce silica structure in silicification. Based on this opinion, in this paper, we employed acidic and basic additives in the formation of LPEI aggregates and focused our attentions to the effect of the additives on silica shape control. The resulting silicas are fruitful in view of shapes of nanofiber-based plates, bundles, flowers, and stick depending upon the additives.

\section{EXPERIMENTAL}

\section{Synthesis of $P-1$}

The linear poly(ethyleneimine) P-1 was prepared by hydrolyzing the commercial poly(2-ethyl-2-oxazoline)s (Polyscience) with $M_{\mathrm{w}} \mathrm{s}$ of 500,000 $\left(M_{\mathrm{w}} / M_{\mathrm{n}}=\right.$ $3.1)$ as follows. Five gram of poly(2-ethyl-2-oxazoline) was dissolved in $90 \mathrm{~mL}$ of $5 \mathrm{M} \mathrm{HCl}$, and the solution was stirred at $90^{\circ} \mathrm{C}$ for $10 \mathrm{~h}$. This hydrolysis gave insoluble white precipitates of hydrochloride salt of P-1. After cooling to room temperature, the solid product $\mathbf{P - 1} / \mathrm{HCl}$ was recovered by filtering and washed three times by acetone. The product $\mathbf{P}-\mathbf{1} / \mathrm{HCl}$ was easily soluble in water, but not in methanol. In ${ }^{1} \mathrm{H}$ NMR $\left(\mathrm{D}_{2} \mathrm{O}\right)$, methyl peak (2.14 ppm) due to acetyl group disappeared and only a single peak at $3.4 \mathrm{ppm}$ due to $\left(-\mathrm{NHCH}_{2} \mathrm{CH}_{2}-\right) / \mathrm{HCl}$ was observed. The obtained solid was dissolved into water and subsequently was charged into a dialysis tube (molecular weight cutoff 3,500 ) and dialyzed against $5 \mathrm{M}$ ammonia water for $3 \mathrm{~d}$ with changing the solution twice every day. This treatment resulted in the free base of P-1 which crystallized in dialysis tube. The crystalline solid was washed by acetone and it was dried at room temperature followed drying under vacuum at $40^{\circ} \mathrm{C}$ for $6 \mathrm{~h}$. Yield, $4.2 \mathrm{~g}$ (hydrated crystalline $\mathbf{P}-\mathbf{1}$ containing about $18 \mathrm{wt} \%$ water estimated from TGA measurement). ${ }^{1} \mathrm{H}$ NMR spectrum (in $\mathrm{D}_{2} \mathrm{O}, 80^{\circ} \mathrm{C}$ ) of the $\mathbf{P}-\mathbf{1}$ (free base form) showed only a single peak at $2.8 \mathrm{ppm}$.

\section{Preparation of P-1 Aggregates}

Additives used in this work include $\mathrm{HCl}$, TSPP, poly(ethylene glycol) bis(carboxymethyl) (BA) and ammonia. For $\mathrm{HCl}$, BAs and ammonia, aqueous solu- tions with desired concentrations of the additives were firstly prepared. Then, P-1 was dissolved in the aqueous solutions of the additives with the desired concentrations at $80^{\circ} \mathrm{C}$. Cooling the hot solutions down to room temperature in air conditions resulted in the aggregates of P-1. For TSPP, the methanol-assisted method was used. First, a methanol solution containing TSPP and P-1 with a desired concentration and molar ratio was prepared by stirring at $80^{\circ} \mathrm{C}$ for $1 \mathrm{~h}$ and stocked at room temperature. Mixing the stock methanol solution and water with suitable ratios resulted in the corresponding aggregates of P-1 incorporated with TSPP.

\section{Silica Deposition and Characterization}

The silica deposition was simply carried out by addition of a silica source into the above $\mathbf{P}-\mathbf{1}$ aggregates solutions. Typically, $1 \mathrm{~mL}$ solution of a $\mathbf{P - 1}$ aggregate was mixed with $2 \mathrm{~mL}$ ethanol solution containing TMOS and ethanol with $1 / 1$ volume ratio. The mixture was left for $40 \mathrm{~min}$ at room temperature. The resulting silica particles were washed by ethanol and centrifuged by $16000 \mathrm{rpm}$ for $5 \mathrm{~min}$. This centrifugation was generally repeated three times. The dried silica particles were viewed by scanning electron microscope (SEM) with Keyence VE7800 microscope.

\section{RESULTS}

\section{$\mathrm{HCl}$ Additive}

As we have shown in our recent work, crystalline hydrogelation of LPEI was sensitively influenced by the additive of $\mathrm{HCl}^{41}$ In general, the completely protonated LPEI is freely soluble in water. However, the partial protonation of LPEI allows the polymer to grow into crystalline in aqueous media. We examined the silicification of TMOS in $1 \% \mathbf{P - 1}$ aggregates prepared at different concentrations of $\mathrm{HCl}$ and investigated the effect of $\mathrm{H}^{+}$addition on silica shape. In the P-1 aggregates prepared from $10^{-5} \mathrm{M} \mathrm{HCl}$ concentration, the silicification from TMOS yielded to loose silica bundles with fibrous structure (Figure 1A). From the magnified image (Figure 1B), it is apparent that many unit nanofibers, which are highly segregated, were spun into the bundles with a network structure. This is sharply contrast with the silica formed without $\mathrm{HCl}$ addition. The silicification in aggregates of $1 \% \mathbf{P - 1}$ assembled from neat water produced very dense silica bundles (Figure 1C). In this case, fibrous structures are observable only at the outside of the bundles (Figure 1D). It is obvious that the slight introduction of $\mathrm{HCl}$ to $\mathbf{P}-\mathbf{1}$ dramatically contributed to the growth of the unit nanofibers with the segregated feature. 

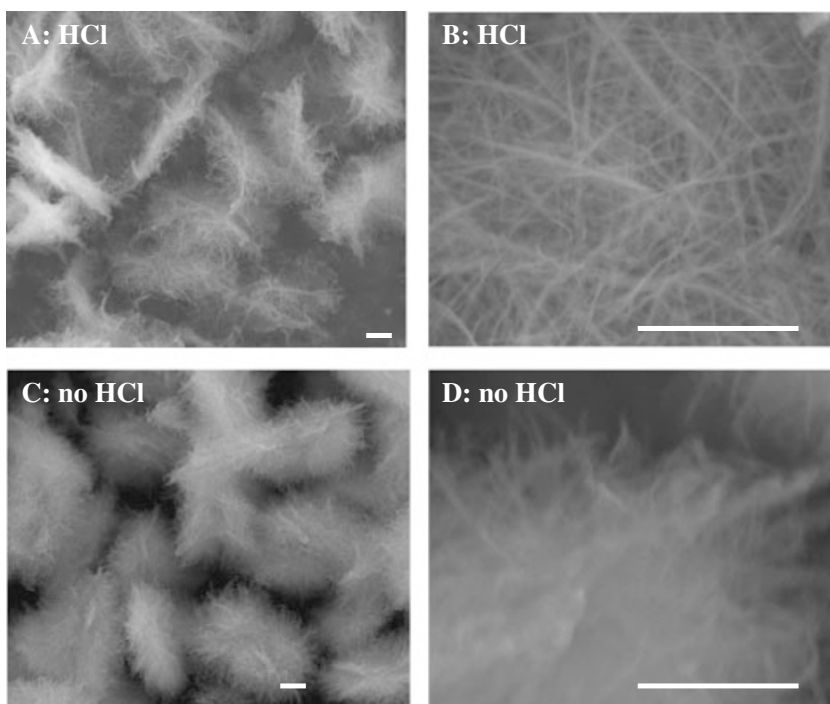

Figure 1. SEM images of P-1-directed silicas with incorporation of $10^{-5} \mathrm{M} \mathrm{HCl}(\mathbf{A}$ and $\mathbf{B})$ and in neat water $(\mathbf{C}$ and $\mathbf{D})$. $\mathbf{B}$ and $\mathbf{D}$ are the higher magnification from $\mathbf{A}$ and $\mathbf{C}$, respectively. The polymer concentration is $1.0 \%$. Bars: $2 \mu \mathrm{m}$.

However, more increasing the concentration of $\mathrm{HCl}$ damaged the nanofiber structure of the resulting silica. Figure 2 shows the SEM images of silicas mediated from P-1 in the presence of higher concentrations of $\mathrm{HCl}$. Fibrous silica structure obtained from the mediation of the $\mathbf{P}-\mathbf{1}$ aggregates prepared in $10^{-3} \mathrm{M} \mathrm{HCl}$ solution became dense (Figure 2A-B) compared to the images seen in Figure $1 \mathrm{C}-\mathrm{D}$, but the bundle shape did not show apparent change. The silica produced in the mediation of the $\mathbf{P} \mathbf{- 1}$ aggregates prepared in $10^{-2} \mathrm{M} \mathrm{HCl}$ solution looked sphere- or olive-like in shape (Figure 2C-D). Interestingly, many silica nanofibers with nearly 1 micrometer length grew from the surface of the spheres, which resemble the image of wheat (Figure 2E). Remarkably, the P-1 aggregates obtained in the suitable concentration of $\mathrm{HCl}$ solutions are capable of directing 3-demensional silica structures consisted of silica nanofibers. In higher $\mathrm{HCl}$ concentration $(0.1 \mathrm{M})$ solution, however, P-1 did not aggregate but gave a transparent solution owing to the heavy protonation of ethyleneimine units (the molar ratio of ethyleneimine unit to $\mathrm{HCl}$ was $\sim 2.3$ ). In this case, no silica deposition occurred even within long time (several hours) after TMOS was added. This is a meaningful clue to understand the template role of the aggregates of $\mathbf{P - 1}$ which are vital in rapid deposition of the shaped silica at ambient conditions. At a condition of further increasing $\mathrm{HCl}$ concentration to $1.0 \mathrm{M}$, many silica particles with irregular shapes were resulted. In this case, the molar ratio of ethyleneimine unit to $\mathrm{HCl}$ was $\sim 0.23$, i.e., $\mathrm{HCl}$ is largely excess. Thus we consider that the irregular silica deposition from TMOS in the medium of
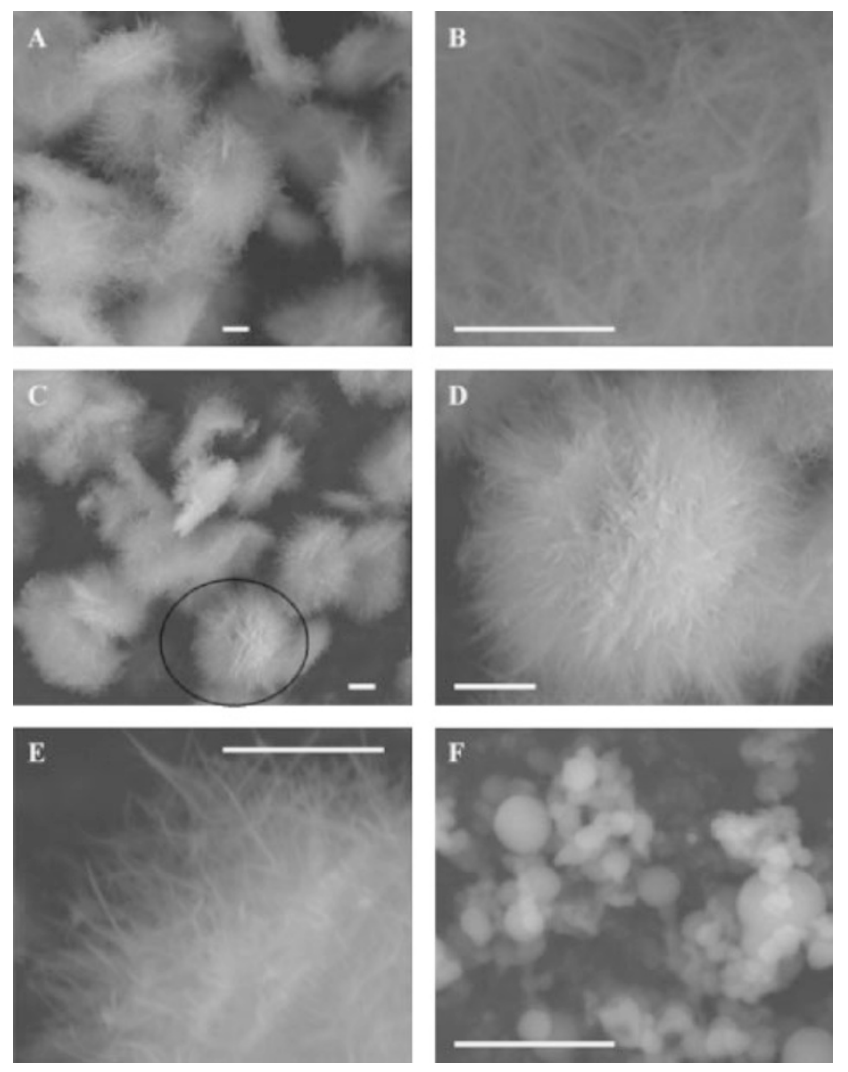

Figure 2. SEM images of silicas mediated by P-1 (1.0\%) with incorporation of $10^{-3} \mathrm{M}$ (A and $\left.\mathbf{B}\right), 10^{-2} \mathrm{M}(\mathbf{C}, \mathbf{D}$ and $\mathbf{E})$, and $1.0 \mathrm{M} \mathrm{HCl}(\mathbf{F})$, respectively. $\mathbf{B}$ and $\mathbf{E}$ are higher magnification from $\mathbf{A}$ and $\mathbf{C}$. D is close observation of circled region in $\mathbf{C}$, showing a typical 3-dimensional silica sphere based on fiber organization. Bars: $2 \mu \mathrm{m}$.

the largely excess of $\mathrm{HCl}$ would be relation with the route of acid catalyzed silicification but not with that of LPEI.

\section{Tetra(4-sulfophenyl)porphyrin (TSPP) Additive Cou- pled with Methanol}

As known above, the acid interacts with P-1 to tune the aggregates of $\mathbf{P - 1}$ and thus influence the silica structures. Organic acids are also promising additives because they can form ionic complexes with LPEI via formation of hydrogen bonding between ethyleneimine units and acidic groups. ${ }^{43-45}$ We have shown that a four-armed star PEI with porphyrin core can direct silica into a beautiful aster in shape, ${ }^{40}$ which is dramatically different to silica mediated from the LPEI. We are sure that the porphyrin residues play an important role in LPEI aggregation. In addition, we have also tried to incorporate a porphyrin possessing sulfonic group such as TSPP with lower polymerization degree (nearly 500) of LPEI for silica shape control.

Employing P-1 which has high polymerization degree nearly to 5050 [estimated from the precursor 

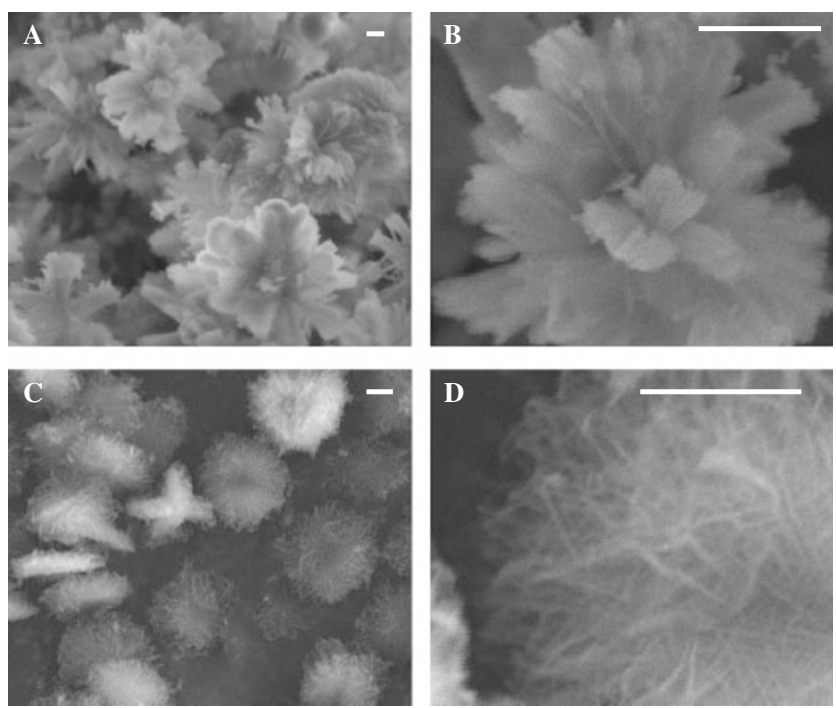

Figure 3. SEM images of silicas prepared from $\mathbf{P - 1}$ with addition of TSPP (EI/TSPP $=1200 / 1$ in molar) $(\mathbf{A}$ and B) and without TSPP (C and D). The aqueous media contains $30 \%$ methanol and $0.3 \% \mathbf{P}-\mathbf{1}$. B and $\mathbf{D}$ are higher magnification from $\mathbf{A}$ and C. Bars $=2 \mu \mathrm{m}$.

poly(2-ethyl-2-oxazoline)], we investigated the effect of the additive of TSPP on silica formation. The silicification performed in an aqueous system containing $30 \%$ methanol, $0.3 \%$ P-1 and trace of TSPP (in a molar ratio of EI/TSPP at 1200/1) produced beautiful flower-like silica particles with micrometer size (Figure 3A). In the flower-like particle, many thicker silica petals with the width of $500-1000 \mathrm{~nm}$ and the length of several micrometers grew from the flower center in a radiation way (Figure 3B). By contrast, in case without addition of TSPP, cake-like silica particles with micrometer sizes were produced (Figure 3C). The cake is composed of many nanofibers (Figure 3D). It seems that the participation of TSPP in the P-1 aggregates efficiently turned the resulting silica from cake-like (2-dimensional) to flower-like (3-dimensional) structure.

\section{Additives of Bifunctional Organic Acids}

It is known from our previous investigation that properties and morphologies of the crystalline aggregates of LPEI were changeable through addition of bifunctional organic acids (BAs), poly(ethylene glycol) bis(carboxymethyl) ethers with molecular weights of ca. 250 and 600 (denoted as BA250 and BA600). ${ }^{41}$ The interactions of BAs with ethyleneimine units provide physical cross-linking via formation of hydrogen bonding. Herein, we examined the influence of the above two acids incorporated with $\mathbf{P - 1}$ on silica morphologies.

The silicification performed in the presence $10^{-5} \mathrm{M}$ of BA600 produced nanofiber-based silica bundles
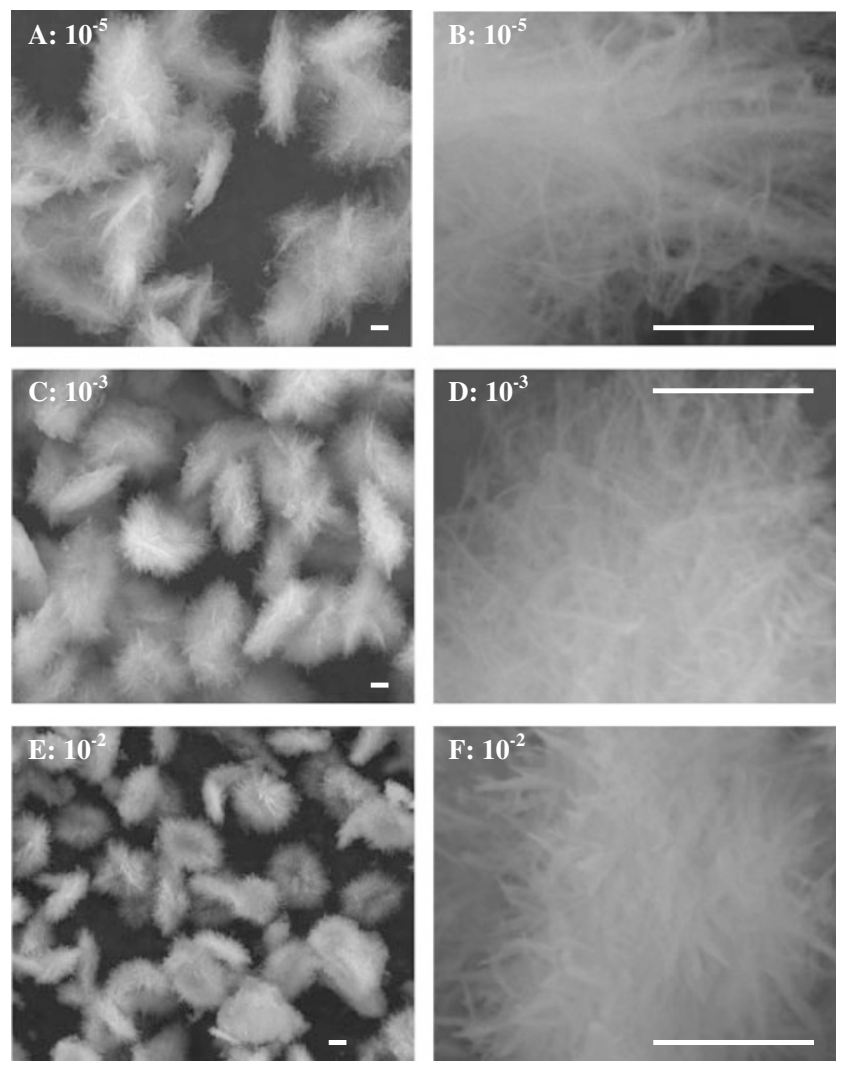

Figure 4. SEM images of P-1-directed silicas by the addition of BA600 with the concentrations of $10^{-5} \mathrm{M}(\mathbf{A}$ and $\mathbf{B}), 10^{-3} \mathrm{M}$ $(\mathbf{C}$ and $\mathbf{D})$ and $10^{-2} \mathrm{M}(\mathbf{E}$ and $\mathbf{F})$. B, D and $\mathbf{F}$ are higher magnification from $\mathbf{A}, \mathbf{C}$ and $\mathbf{E}$. The polymer concentration is $1.0 \%$. Bars $=2 \mu \mathrm{m}$.

with relatively loose and expanding structure (see Figure 4A-B). This is very similar to the silica mediated from weakly protonated $\mathbf{P - 1}$ aggregates seen in Figure 1A-B. The system with $10^{-4} \mathrm{M}$ BA600 gave the same silica (data not show) to that from $10^{-5} \mathrm{M}$ concentration. However, as can be seen in Figure 4C-D, the silica obtained at $10^{-3} \mathrm{M}$ BA600 changed slightly in its size and shape, for example, the silica bundles became small and dense. When the concentration of BA600 was raised to $10^{-2} \mathrm{M}$, the silicification produced smaller silica bundles that are nearly half of the former in size. This tendency of bundle-size decreasing is in agreement with the case of crystalline aggregates of LPEI; the size of the aggregates decreased with increasing the BAs concentrations. ${ }^{41}$ Nevertheless the silica bundles became smaller, fibrous structures is still remained on the surface of the bundle silica (Figure 4E-F).

The silica deposition reaction was also performed using BA250, which possesses relatively shorter spacer than BA600 between the two acid groups. The silica mediated from the lower concentrations of BA250 $\left(10^{-5}\right.$ and $\left.10^{-4} \mathrm{M}\right)$ has nearly same images (data not shown) to that from $10^{-3} \mathrm{M}$ of BA600. 

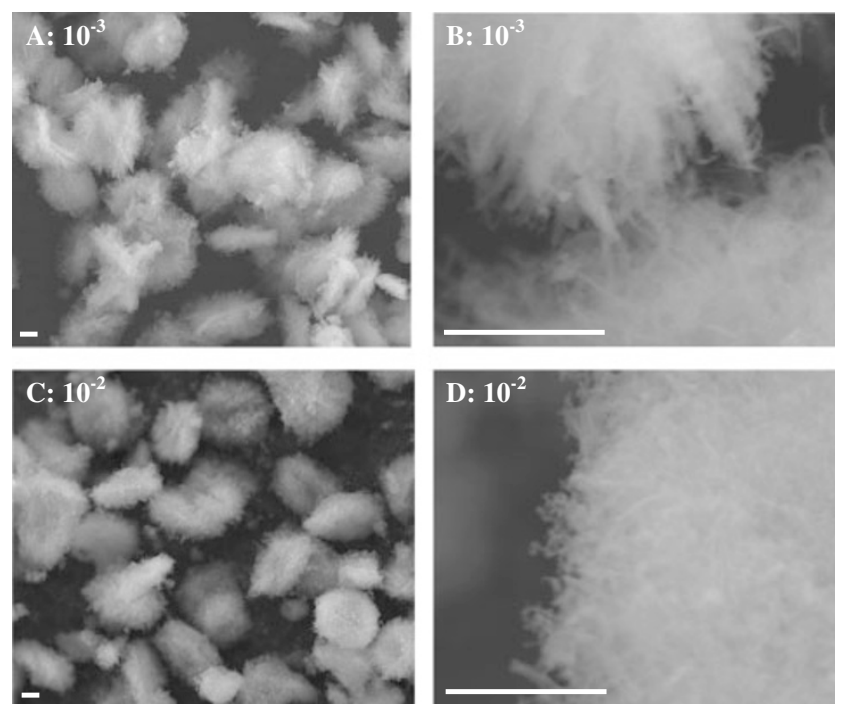

Figure 5. SEM images of P-1-directed silicas in the presence of BA250 with the concentrations of $10^{-3} \mathrm{M}(\mathbf{A}$ and $\mathbf{B})$, and $10^{-2}$ $\mathbf{M}(\mathbf{C}$ and $\mathbf{D})$. $\mathbf{B}$ and $\mathbf{D}$ are higher magnification from $\mathbf{A}$ and $\mathbf{C}$. The polymer concentration is $1.0 \%$. Bars $=2 \mu \mathrm{m}$.

However, the SEM images of the silica particles obtained in the $10^{-3}$ and $10^{-2} \mathrm{M}$ concentrations of BA250 are remarkably different compared to the silica formed from the same concentrations of BA600. As shown in Figure 5, these silica particles looked much denser (Figure 5A-D) in comparison with the silica mediated by P-1/BA600 association (Figure 4B-F) and almost no fibrous structure was observed from the silica particles mediated from the higher concentration $\left(10^{-2} \mathrm{M}\right)$ of BA250 (Figure 5D).

\section{Ammonia Additive}

In ammonia medium, LPEI forms easily into crystalline aggregate even at very low concentration of LPEI. ${ }^{41}$ We performed silica deposition in the mediation of $1 \% \mathbf{P - 1}$ aggregates prepared at different concentrations of ammonia solution. Figure 6 shows typical SEM images of the resulting silica. It is observable that the silica mediated by the $\mathbf{P - 1}$ aggregates prepared in $10^{-5} \mathrm{M}$ ammonia solution did not show notable change in their shape (Figure 6A) compared to the silica from neat water (Figure 1C-D). By the aggregates prepared in higher ammonia concentration $(1.0 \mathrm{M})$, we obtained thin and long silica bundles that are like sticks piled randomly (Figure 6B). In the magnified image, it can be seen that the bundles were bridged by some of nanofibers separated very well (Figure 6C). Further increase of ammonia concentration to $6.0 \mathrm{M}$ resulted in a mixture of bundles and particulates. It is clear that the bundle is dense and some longer nano fibers attached on the main bundle (Figure 6D).
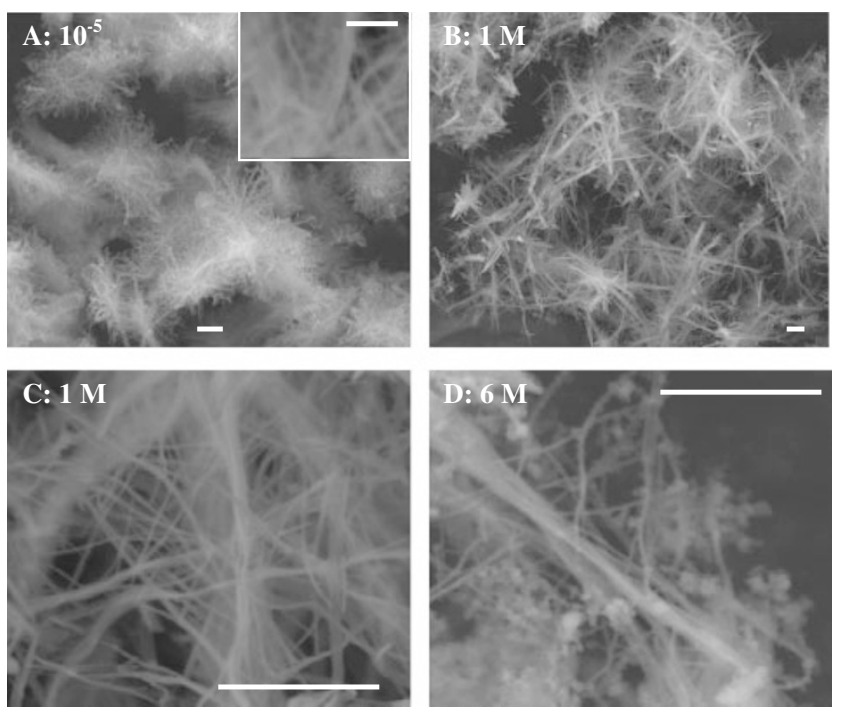

Figure 6. SEM images of P-1-directed silicas in the presence of ammonia with the concentrations of $10^{-5} \mathrm{M}(\mathbf{A}), 1.0 \mathrm{M}$ (B and C) and $6.0 \mathrm{M}(\mathbf{D})$. The polymer concentration is $1.0 \%$. Bars $=2$ $\mu \mathrm{m}$ and inset bar $=500 \mathrm{~nm}$.

Table I. Silica mediated by P-1 aggregates incorporated with additives

\begin{tabular}{ll}
\hline Additive & Silica morphology \\
\hline $\mathrm{HCl}\left(10^{-5} \mathrm{M}\right.$ in water $)$ & fibrous network plate \\
$\mathrm{NH}_{3}(1 \mathrm{M}$ in water $)$ & straight silica bundles \\
$\mathrm{BS} 250\left(10^{-2} \mathrm{M}\right.$ in water $)$ & dense and small particles \\
$\mathrm{BS} 600\left(10^{-2} \mathrm{M}\right.$ in water $)$ & fibrous particles \\
$\mathrm{TSPP}$ (trace in water/methanol $)$ & flower like particles \\
\hline
\end{tabular}

\section{DISCUSSION}

Linear poly(ethyleneimine) has strong tendency to form fibrous crystalline aggregates in aqueous media. This feature is a crucial reason why shaped silica forms in the LPEI system. A model of LPEI aggregates with a rod like core-brush micelle was proposed for explaining the silica fiber formation. ${ }^{39,41}$ In this model, the LPEI brush which attached on the fibrous core of LPEI crystalline just plays not only the role of scaffold to enrich silica precursor around the core but also the role of catalyst to accelerate the hydrolytic condensation of the enriched precursor forming silica shell. The brush quantity would contribute the stabilization of the fibrous aggregates of LPEI, and the quantity balance between the brush-LPEI and the core-LPEI would determine the silica nanofiber formation and growth.

The major influences of acidic/basic additives on the silica formation are summarized in Table I. In the mediation by $10^{-5} \mathrm{M} \mathrm{HCl}$, the molar ratio of ethyleneimine units to $\mathrm{HCl}$ is nearly $23000 / 1$. We think that, in this ratio, the residue of $\left(\mathrm{NHCH}_{2} \mathrm{CH}_{2}\right)$ in brush 
part will bear enough cationic charges, and thus be favorable to cause the segregation of the fibrous aggregates due to the electrostatic and steric repulsion (i.e., electrosteric stabilization) between the brushes. ${ }^{46}$ This is a considerable reason why the silica fiber produced in the presence of a trace of $\mathrm{HCl}$ takes in the well-segregated state than that obtained from neat water. ${ }^{39}$

The introduction of TSPP into $\mathbf{P}-\mathbf{1}$ can efficiently adjust polymer organization and direct subsequently the silica shapes and structures. It should be noted that the existence of methanol in aqueous media retards generally the formation of the crystalline P-1, for example P-1 cannot aggregate at higher methanol contents. $^{40,42}$ At the same time, TSPP also disturbs the P-1 crystallization due to providing physical crosslinking via hydrogen bonding formation between $-\mathrm{SO}_{3} \mathrm{H}$ and $-\mathrm{NH}-$. However, these interactions can promote P-1 to form subtle aggregates different to the crystalline organization. The result of the shift of the silica structures from the thinner nanofibers to the thicker petals (Figure 3) would also be related to the retarding of P-1 crystalline due to TSPP addition. Meanwhile, the flower structure formation should be attributed to the stacking interactions of the porphyrin residues that anchored to the $\mathbf{P}-\mathbf{1}$ chains with hydrogen bonding. In comparison, the contribution of bifunctional organic acids, such as BA250 and BA600, appeared mainly on the control of the size and density of the silica bundles (Figures 4 and 5). They did not change the morphology of the resulting silica. Therefore, we can conclude that in the silicification mediated by aggregates of LPEI, the additives only providing cross-linking sites are not promising for directing precise silica structures. Hydrogen bond cross-linking with coupling additional interactions such as seen in TSPP additive, which not only supplies cross-linking sites towards LPEI but also causes additional $\pi-\pi$ interactions by itself, would have great potential to direct silica morphology.

Increasing ammonia concentrations in $\mathbf{P}-\mathbf{1}$ aggregation allows P-1 to grow with the straightly elongated fiber structures, in which some fibers probably closed together and some ones separated each other. The silica deposition occurred in the closed regions would result in bundles structure, and the silica nucleated in the separated fibrous zone would grow into long silica fiber (Figure 6). At high concentration of ammonia, the resulting silica consisted of both bundles and non-structured particulates (see Figure 6D). The formation of the silica particulates must be related to the catalysis role of ammonia ${ }^{47}$ that accelerates the hydrolytic condensation of TMOS to yield non-ordered silica. In the case of ammonia addition, it is interesting that the two routes of silica formations, one from P-1 directed fashion and the other from ammonia catalyzed route, seem not to interfere mutually. The two routes could be attributed to the striking difference of silica deposition mechanisms. P-1 fibers in ammonia medium serve template for the silica deposition under ambient conditions to give controlled silica. However, the hydrolytic condensation of TMOS catalyzed by ammonia offers the silica without structural directions.

\section{CONCLUSIONS}

We have successfully demonstrated that multiply shaped silicas were available through silicification in the P-1/aqueous systems incorporated with various proton related additives. The resulting silicas with different shapes and structures were controllable with changing acidic/basic additives. This approach by the additives with incorporation of simple LPEI to shape-controlled silicas is of particular importance since this route does not need the complex LPEI polymer architectures that are time-consuming and highcost. We believe that many organic and inorganic compounds, which are capable of interacting with LPEI, are applicable for directing silica structures and developing novel silica materials.

\section{REFERENCES}

1. S. Mann, J. Webb, and R. J. P. Williams, "Biomineralization," VCH, Weiheim, 1989.

2. E. Bauerlein, "Biomineralization," Wiley-VCH, Weiheim, 2000 .

3. E. Bauerlein, Angrew. Chem., Int. Ed., 42, 614 (2003).

4. S. Mann, "Biomimetic Materials Chemistry," VCHWeiheim, New York, 1996.

5. S. H. Yu and H. Colfen, J. Mater. Chem., 14, 2124 (2004).

6. S. Zhang, Nat. Biotechnol., 21, 1171 (2003).

7. D. E. Davis, Nature, 417, 813 (2002).

8. C. T. Kresge, M. E. Leonowicz, W. J. Roth, J. C. Vartuli, and J. S. Beck, Nature, 359, 710 (1992).

9. D. Y. Zhao, J. Feng, Q. Huo, N. Melosh, G. H. Fredrickson, B. F. Chmelka, and G. D. Stucky, Science, 279, 548 (1998).

10. S. Shinkai, Angew. Chem., Int. Ed., 42, 614 (2003).

11. H. Yang, N. Coombs, and G. A. Ozin, Nature, 386, 692 (1997).

12. G. A. Ozin, Acc. Chem. Res., 30, 17 (1997).

13. I. Sokolov, H. Yang, G. A. Ozin, and C. Y. Kresge, Adv. Mater., 11, 636 (1999).

14. J. Wang. C. K. Tsung, R. C. Hayward, Y. Wu, and G. D. Stucky, Angew. Chem., Int. Ed., 44, 332 (2005) and references therein.

15. W. E. G. Muller, "Silicon Biomineralization: BiologyBiochemistry-Molecular Biology-Biotechnology," Springer, Berlin, 2003.

16. M. Hildebrand, Prog. Org. Coat., 7, 256 (2003).

17. D. E. Morse, Trends Biotechnol., 17, 230 (1999). 
18. a) C. W. P. Foo, J. Huang, and D. L. Kaplan, Trends Biotechnol., 22, 577 (2004).

b) M. Sumper and N. Kroger, J. Mater. Chem., 14, 2059 (2004).

19. K. Shimizu, J. Cha, G. D. Stucky, and D. E. Morse, Proc. Natl. Acad. Sci. U.S.A., 95, 6234 (1998).

20. N. Kröger, R. Deutzmann, and M. Sumper, Science, 286, 1129 (1999).

21. N. Poulsen, M. Sumper, and N. Kröger, Proc. Natl. Acad. Sci. U.S.A., 100, 12075 (2003).

22. J. Cha, G. D. Stucky, and D. E. Morse, Proc. Natl. Acad. Sci. U.S.A., 96, 361 (1999).

23. M. R. Knecht and D. W. Wright, Chem. Commun., 3038 (2003).

24. T. Coradin, O. Durupthy, and J. Livage, Langmuir, 18, 2331 (2002).

25. S. V. Patwardhan and S. J. Clarson, Silicon Chem., 1, 207 (2002).

26. M. Sumper, S. Lorenz, and E. Brunner, Angew. Chem., Int. Ed., 42, 5192 (2003).

27. E. G. Vrieling, T. P. M. Beelen, R. A. van Santen, and W. W. C. Bieskes, Angew. Chem., Int. Ed., 41, 1543 (2002).

28. M. R. Knecht and D. W. Wright, Langmuir, 20, 4728 (2004).

29. H. Menzel, S. Horstmann, P. Behrens, P. Barnreuther, I. Krueger, and M. Jahns, Chem. Commun., 2994 (2003).

30. K. M. Roth, Y. Zhou, W. Yang, and D. E. Morse, J. Am. Chem. Soc., 127, 325 (2005).

31. D. Belton, G. Paine, S. V. Patwardhan, and C. C. Perry, J. Mater. Chem., 14, 2231 (2004).

32. T. Coradin, C. Roux, and J. Livage, J. Mater. Chem., 12,
1242 (2002).

33. J. N. Cha, G. D. Stucky, D. E. Morse, and T. J. Deming, Nature, 403, 289 (2000).

34. F. Rodriguez, D. D. Glawe, R. R. Naik, K. P. Hallinan, and M. O. Stone, Biomacromolecules, 5, 261 (2004).

35. R. R. Naik, P. W. Whitlock, F. Rodriguez, L. L. Brott, D. D. Glawe, S. J. Clarson, and M. O. Stone, Chem. Commun., 238 (2003).

36. R. R. Naik, L. L. Brott, F. Rodriguez, G. Agarwal, S. M. Kirkpatrick, and M. O. Stone, Prog. Org. Coat., 47, 249 (2003).

37. S. V. Patwardhan, N. Mukherjee, M. Steinitz-Kannan, and S. J. Clarson, Chem. Commun., 1122 (2003).

38. M. Sumper, Angew. Chem., Int. Ed., 43, 2251 (2004).

39. R.-H. Jin and J.-J. Yuan, Chem. Commun., 1399 (2005).

40. J.-J. Yuan and R.-H. Jin, Adv. Mater., 17, 885 (2005).

41. a) J.-J. Yuan and R.-H. Jin, Langmuir, 21, 3136 (2005). b) J.-J. Yuan, P.-X. Zhu, N. Fukazawa, and R.-H. Jin, Adv. Fucnt. Mater., 16, 2205 (2006).

42. a) R.-H. Jin and J.-J. Yuan, Macromol. Chem. Phys., 206, 2160 (2005).

b) R.-H. Jin and J.-J. Yuan, Chem. Mater., 18, 3390 (2006).

43. H.-L. Chen and M.-S. Hsiao, Macromolecules, 32, 2967 (1999).

44. B. Ren, Z. Tong, X. Liu, C. Wang, and F. Zeng, Langmuir, 20, 10737 (2004).

45. A. F. Thunemann and S. General, Macromolecules, 34, 6978 (2001).

46. X. Guo and M. Ballauff, Langmuir, 16, 8719 (2000).

47. R. K. Iler, "The Chemistry of Silica," Wiley, New York, 1979. 